\title{
CONCERNING POINT SETS WHICH CAN BE MADE CONNECTED BY THE ADDITION OF A SIMPLE CONTINUOUS ARC*
}

BY

\section{GORDON T. WHYBURN}

In their paper On the most general plane closed point set through which it is possible to pass a simple continuous arc, R. L. Moore and J. R. Kline† prove that it is possible to pass a simple continuous arc through every closed and bounded set $M$ having the property that every closed and connected subset of $M$ is either a single point or an $\operatorname{arc} t$ such that no point of $t$, with the exception of its end points, is a limit point of $M-t$. It is clear, however, that in order that a simple continuous arc may be drawn in such a way as to contain at least one point of every maximal connected subset of a point set $M$, it is not necessary that the set $M$ be of the particular type satisfying their theorem. In this paper I shall make a study of certain conditions which a point set must satisfy in order that a simple continuous arc or an open curve may be drawn in such a way that the set in question plus that arc or curve will be connected.

LEMмA I. If $M$ is any closed and bounded point set, then there exists a countable number of arcs $t_{1}, t_{2}, t_{3}, \cdots$, such that for every positive integer $n, t_{n}$ contains at least one point of every maximal connected subset of $M$ which is of diameter greater than $1 / n$.

Let $n$ denote any definite positive integer. Since $M$ is bounded, there exists a square $S$ which encloses $M ; S$ plus its interior can be divided by a finite number of straight lines parallel and perpendicular to the bases of $S$ into a finite number of squares plus their interiors in such a way that the diameter of each of these squares is less than $1 / n$ and such that the interiors of no two of them have a point in common. Let $G$ denote this finite set of squares (not including their interiors), and let $T$ denote the point set obtained by adding together all the point sets of the set $G$. Then since the interior of every square of the set $G$ is of diameter less than $1 / n$, every maximal connected subset of $M$ which is of diameter greater than $1 / n$ must contain at least one point in common with $T$. Let $F$ denote the set of all points common to $M$ and $T$. From each maximal connected subset $Y$ of $F$ select exactly one point

* Presented to the Society, April 2, 1926; received by the editors in April, 1926.

† Annals of Mathematics, (2), vol. 20 (1919), pp. 218-223. 
$X$; and let $P$ denote the set of all such points $(X)$ thus selected. Then since $F$ is closed and has no continuum of condensation, it follows that $\bar{P}$ is a closed and totally disconnected point set.* It follows, then, from the above mentioned theorem of Moore and Kline $\dagger$ that $\bar{P}$ is a subset of a simple continuous arc $t_{n}$. Clearly $t_{n}$ contains at least one point of every maximal connected subset of $M$ which is of diameter greater than $1 / n$.

THEOREM 1. If $M$ is a closed and bounded point set, a necessary and sufficient condition that there should exist a simple continuous arc which contains at least one point of every maximal connected subset of $M$ is that for every continuum $K$ of $M$ which consists of more than one point there should exist a positive number $\epsilon_{k}$ such that $K$ is not the limiting set of any collection of maximal connected subsets of $M$ each of diameter less than $\epsilon_{k}$.

(I). The condition is sufficient. For since $M$ satisfies the conditions of Lemma I, there exists a countable set of $\operatorname{arcs} t_{1}, t_{2}, t_{3}, \cdots$, having the same property with respect to $M$ that the corresponding set of arcs in Lemma I has with respect to the set $M$ of Lemma I. Let $K_{1}$ denote the set of points common to $t_{1}$ and $M$; let $K_{2}$ denote the set common to $t_{2}$ and to those maximal connected subsets of $M$ which have no point in $t_{1} ; K_{3}$ the set common to $t_{3}$ and to those maximal connected subsets of $M$ which have no point in $t_{1}+t_{2}$; in general, let $K_{n}$ denote the set of points common to $t_{n}$ and to those maximal connected subsets of $M$ which have no point in $t_{1}+t_{2}+t_{3}+t_{4}+\cdots+t_{n-1}$. Let $K$ denote the point set $K_{1}+K_{2}+K_{3}+K_{4}+\cdots$. I will proceed to show that $\bar{K}$ contains no continuum of condensation. Suppose, on the contrary, that $\bar{K}$ contains a continuum of condensation $H$. Then $H$ is also a continuum of condensation of $M$; and by hypothesis there exists a positive number $\epsilon_{H}$ such that $H$ is not the limiting set of any set of maximal connected subsets of $M$ each of diameter less than $\epsilon_{H}$. Now the elements of $K$ have been so selected that for any given positive number, say $\epsilon_{H}$, there exists a positive number $\delta\left(\epsilon_{H}\right)$ such that for every integer $n>\delta\left(\epsilon_{H}\right), K_{n}$ contains points of only those maximal connected subsets of $M$ which are of diameter less than $\boldsymbol{\epsilon}_{\boldsymbol{H}}$. Let $i$ denote an integer greater than $\delta\left(\epsilon_{H}\right)$. Then $\sum_{n=i+1}^{n=\infty} K_{n}$ contains points of only those maximal connected subsets of $M$ which are of diameter less than $\epsilon_{H}$. It follows that not every point of $H$ is a limit point of $\sum_{\substack{n=i+1 \\ n=i}}^{n} K_{n}$. Let $G$ denote the collection of point sets $K_{1}, K_{2}, K_{3}, \cdots, K_{i}$. Let

$$
A=\sum_{n=1}^{n=i} K_{n} \text {, and let } B=\sum_{n=i+1}^{n=\infty} K_{n} \text {. }
$$

* In this paper wherever a symbol $X$ is used to denote a point set, the symbol $\bar{X}$ will be used to denote the set $X$ plus all those points which are limit points of $X$.

† R. L. Moore and J. R. Kline, loc. cit. 
Then $K=A+B$. Let $P$ denote a point of $H$ which is not a limit point of $B$; and let $C$ be a circle enclosing $P$ and not enclosing or containing any point whatever of $\bar{B}$. From a theorem due to Janiszewski, ${ }^{*}$ it follows that $C$ plus its interior contains a subcontinuum $D$ of $H$. Then $D$ is a subset of the closed set $\bar{A}$. Let $K_{a}$ and $K_{b}$ denote any two elements of $G, K_{a}$ denoting the one of lower subscript. I will show that $\bar{K}_{a}$ and $\bar{K}_{b}$ have at most a closed and totally disconnected set in common. Suppose, on the contrary, that $\bar{K}_{a}$ and $\bar{K}_{b}$ have in common a continuum $t$ which consists of more than one point. Then $t$ is a subset both of $t_{a}$ and of $t_{b}$; hence $t$ is an arc. Let $E$ and $F$ denote the end points of $t$. Since $t$ is a subset of $t_{a}$, and since $t_{a}$ precedes $t_{b}$, then no point of $t$ can belong to $K_{b}$. And since no point of $t$ except the points $E$ and $F$ can be a limit point of $t_{b}-t$, then no points of $t$ except $E$ and $F$ can belong to $\bar{K}_{b}$. But by supposition, $t$ is a subset of $\bar{K}_{b}$. It follows that $\bar{K}_{a}$ and $\bar{K}_{b}$ have at most a closed and totally disconnected set in common. Let $U$ denote the set of all points $(X)$ of $\bar{A}$ such that for some two elements $K_{a}$ and $K_{b}$ of $G, X$ is common to $\bar{K}_{a}$ and $\bar{K}_{b}$. Since $U$ is the sum of a finite number of closed and totally disconnected point sets, $U$ itself must be closed and totally disconnected. Hence $D$, a continuum consisting of more than one point, cannot be a subset of $U$. Therefore, there exists a point $P$ of $D$ such that for some element $K_{p}$ of $G, P$ belongs to $\bar{K}_{p}$ and is not a limit point of $\bar{A}-\bar{K}_{p}$. It follows from the above mentioned theorem of Janiszewski's $\dagger$ that $\bar{K}_{p}$ contains a continuum $l$ of $D$ such that no point of $l$ is a limit point of $\bar{A}-\bar{K}_{p}$. But $l$ is a subset of $t_{p}$. Hence $l$ is an arc, and no points of $l$ except its end points can be limit points of $\bar{K}_{p}-l$. Hence if $O$ is an interior point of $l, O$ is not a limit point of $\bar{K}-l$. But $l$, by supposition, belongs to $H$, a continuum of condensation of $\bar{K}$. Thus the supposition that $\bar{K}$ contains a continuum of condensation leads to a contradiction.

Now from each maximal connected subset $Y$ of $\bar{K}$ let us select exactly one point $X$. Let $N$ denote the set of all the points $(X)$ thus selected. Since $\bar{K}$ contains no continuum of condensation, it readily follows that $\bar{N}$ is a closed and totally disconnected set. It is clear that $\bar{N}$ contains at least one point of every maximal connected subset of $M$ which is of diameter greater than 0 . Let $Q$ denote the set of all those maximal connected subsets of $M$ which have no point in common with $\bar{N}$. Then since every maximal connected subset of $Q$ is a single point, it follows from our hypothesis that $\bar{Q}$ is a closed and totally disconnected point set. Let $R$ denote the point set $\bar{N}+\bar{Q}$.

\footnotetext{
* Sur les continus irréductibles entre deux points, Journal de l'Ecole Polytechnique, (2), vol. 16 (1912), p. 109.

$\dagger$ Loc. cit.
} 
Clearly $R$ is closed and totally disconnected; accordingly, there exists a simple continuous arc $T_{0}$ which contains $R ; T_{0}$ contains at least one point of every maximal connected subset of $M$.

(II). The condition is also necessary. Suppose, on the contrary, that there exists a closed and bounded point set $M$ and a simple continuous arc $T$ such that $T$ contains at least one point of every maximal connected subset of $M$, but such that $M$ does not satisfy the condition of Theorem 1 . Then $M$ contains some continuum $K$ consisting of more than one point and such that for every positive number $\epsilon, K$ is the limiting set of a set of maximal connected subsets of $M$ each of diameter less than $\epsilon$. I will show that every point of $K$ must be a limit point of $T-K \cdot T$. For suppose $K$ contains a point $P$ which is not a limit point of $T-K \cdot T$. Let $C$ be a circle having $P$ as center and not enclosing any point of $T-K \cdot T$ and of radius less than $\frac{1}{3}$ of the diameter of $K$. Let $r$ denote the radius of $C$. By hypothesis there exists a set $L$ of maximal connected subsets of $M$ each of which is of diameter less than $\frac{1}{4} r$ such that $K$ is the limiting set of $L$. Since $P$ belongs to $K$, there exists an element $g$ of $L$ which contains a point whose distance from $P$ is less than $\frac{1}{4} r$; and since $g$ is of diameter less than $\frac{1}{4} r, g$ must lie wholly within $C$. But $g$ must contain at least one point $Q$ of $T$. Now since $K$ is of diameter $\geqq 3 r, K$ cannot be an element of $L$. Hence $Q$ does not belong to $K$, and therefore must belong to $T-K \cdot T$. But $Q$ lies within $C$, and $C$, by supposition, encloses no point of $T-K \cdot T$. It follows, then, that every point of $K$ is a limit point of $T-K \cdot T$. It is easily seen that $K$ must be a subset of $T$; and since $K$ is closed and connected and consists of more than one point, $K$ must be an arc. And if $O$ denotes an interior point of $K$, then $O$ is not a limit point of $T-K$. But we have just shown that every point of $K$ is a limit point of $T-K$. Thus the hypothesis that the condition of Theorem 1 is not necessary leads to a contradiction, and the theorem is proved.

Definition. A point set $M$ will be said to satisfy Condition $\mathrm{L}$ provided it is true that if $K$ is any continuum whatever consisting of more than a single point, then there exists a positive number $\epsilon_{K}$ such that $K$ is not a subset of the limiting set of any collection of maximal connected subsets of $M$ each of diameter less than $\epsilon_{K}$.

THEOREM 2. If $M$ is any closed point set, then in order that there should exist a simple continuous arc which contains at least one point of every maximal connected subset of $M$ it is necessary and sufficient (1) that there should exist a bounded portion of the plane which contains at least one point of every maximal connected subset of $M$, and (2) that $M$ should satisfy Condition $\mathrm{L}$. 
It follows by an argument similar to part (II) of the proof of Theorem 1 that the conditions are necessary. I will proceed to show that they are sufficient. By hypothesis it follows that there exists a circle $C$ such that $C$ plus its interior contains at least one point of every maximal connected subset of $M$. Let $R$ denote the interior of $C$, and let $N$ denote the set of points common to $M$ and to $R+C$. It readily follows that $N$ satisfies Condition $\mathrm{L}$; and since $N$ is closed and bounded, it follows from Theorem 1 that there exists an arc $T$ which contains at least one point of every maximal connected subset of $N$. But every maximal connected subset of $N$ belongs to a single maximal connected subset of $M$, and each maximal connected subset of $M$ contains at least one maximal connected subset of $N$. If follows, then, that $T$ contains at least one point of every maximal connected subset of $M$.

TheOREM 3. If $M$ is a closed point set which satisfies conditions (1) and (2) of Theorem 2, and if $K$ is a closed and bounded subset of $M$ having the property that every subcontinuum of $K$ is either a single point or an arc $t$ such that no point of $t$, with the exception of its end points, is a limit point of $M-t$, then there exists an arc $T$ which contains $K$ and which contains at least one point of every maximal connected subset of $M$.

By an argument almost identical with part (I) of the proof of Theorem 1, it follows that there exists a closed, bounded, and totally disconnected point set $R$ which contains at least one point of every maximal connected subset of $M$. Let $N$ denote the point set $K+R$. Then clearly $N$ satisfies all the conditions of the above mentioned theorem of Moore and Kline.* Accordingly, there exists a simple continuous arc $T$ which contains $N$; $T$, then, contains $K$ and also contains at least one point of every maximal connected subset of $M$.

It is interesting to note that Theorem 3 is a generalization of Moore and Kline's theorem. It reduces to their theorem in case $K=M$.

Theorem 4. If $M$ is a bounded point set such that the totality of all those limit points of $M$ which do not belong to $M$ is a closed set, then in order that there should exist a simple continuous arc which contains at least one point of every maximal connected subset of $M$ it is necessary and sufficient that $M$ should satisfy Condition L.

That the condition is necessary follows by an argument identical with part (II) of the proof of Theorem 1. I shall show that the condition is sufficient. Let $M^{\prime}$ denote the totality of all those limit points of $M$ which $M$

* Loc. cit. 
does not contain. For any definite positive integer $n$, let the sets $S, G, T, F$, and $P$ be selected exactly as was done in the proof of Lemma $I$. Then $\bar{P}$ is totally disconnected. For suppose $\bar{P}$ contains a continuum $H$ consisting of more than a single point. Then every point of $H$ is a limit point of a set of points of $P$ which belong to $H$. And since $M^{\prime}$ is closed, it readily follows from Janiszewski's theorem mentioned above that $H$ contains a continuum $D$ which consists of more than one point and which is a subset of $M$. Since $D$ is a subset of a finite number of arcs, then $D$ must contain at least one $\operatorname{arc} t$ such that only the end points of $t$ are limit points of $T-t$. But since $t$ belongs to only one maximal connected subset of $F$, then $P$ contains only one point at most of $t$. And since only the end points of $t$ can be limit points of $P$, $\bar{P}$ can contain at most three points of $t$. Thus the supposition that $\bar{P}$ is not totally disconnected leads to a contradiction. It follows, then, that there exists a simple continuous arc $t_{n}$ which contains $\bar{P}$, and therefore contains at least one point of every maximal connected subset of $M$ which is of diameter greater than $1 / n$. Hence, there exists a countable set of $\operatorname{arcs} t_{1}, t_{2}, t_{3}, \cdots$, such that for every positive integer $n, t_{n}$ contains at least one point of every maximal connected subset of $M$ of diameter greater than $1 / n$.

Now let the sets $K_{1}, K_{2}, K_{3}, \cdots, K, N$, and $R$ be selected exactly as in the proof of Theorem 1 . It can then be shown that $R$ is totally disconnected. For suppose $R$ contains a continuum $H$ consisting of more than one point. Then either (1) $H$ belongs wholly to $M^{\prime}$, or (2) $H$ contains a subcontinuum $D$ which belongs wholly to $M$ and which consists of more than a single point. In either case, $H$ is a continuum of condensation of $\bar{K}$, and either of the two cases can be shown to lead to a contradiction by the same method as was used in the proof of Theorem 1 to show that the set $\bar{K}$ contained no continuum of condensation. It follows, then, that $R$ is closed and totally disconnected; consequently, there exists a simple continuous arc which contains $R$ and which therefore contains at least one point of every maximal connected subset of $M$.

THEOREM 5. In order that a closed point set $M$ (which is not itself an open curve) should be a subset of an open curve, it is necessary and sufficient (1) that every subcontinuum of $M$ should be either a single point or a set $t$ such that $t$ is either an arc or a ray of an open curve having the property that no point of $t$, with the exception of its end point $(s)$, is a limit point of $M-t$, and (2) that if $M$ contains two rays $r_{1}$ and $r_{2}$, then $M-\left(r_{1}+r_{2}\right)$ is a bounded point set.

The conditions are evidently necessary. I shall show that they are suffcient. There exists a circle $C$ with center $O$ such that $C$ plus its interior contains no point of $M$. By an inversion of the whole plane about the circle 
$C, M$ is thrown into a bounded point set $M^{*}$ which is closed except possibly for the point $O$. It is easily shown that the image under this inversion of every $\operatorname{arc} t$ of $M$ is an $\operatorname{arc} t^{*}$ of $M^{*}$, and that the image of every ray $r$ of $M$ is an arc minus one end point in $M^{*}$, that end point in every case being the point $O$ itself. Since $M$ contains not more than two mutually exclusive rays, then $O$ is an end point of not more than two arcs of $M^{*}+O$ which have in common only the point $O$; and if $O$ is an end point of two such arcs, i.e., if $O$ is an interior point of any arc of $M^{*}+O$, then $O$ is not a limit point of $M^{*}+O$ minus that maximal connected subset of $M^{*}+O$ to which $O$ belongs. It readily follows, then, that $M^{*}+O$ is a closed and bounded point set which satisfies all the conditions of Moore and Kline's theorem quoted above. Accordingly, there exists a simple continuous arc $t$ which contains $M^{*}+O$. Let $A$ and $B$ denote the extremities of $t$. There exists an $\operatorname{arc} t_{0}$ from $A$ to $B$ having only the points $A$ and $B$ in common with $t$. Let $l^{*}$ denote the simple closed curve $t+t_{0}$. It can easily be shown that the point set of which $l^{*}-O$ is the image is an open curve which contains $M$.

Theorem 6. If $M$ is a closed point set (bounded or not), then in order that there should exist an open curve which contains at least one point of every maximal connected subset of $M$ it is necessary and sufficient that $M$ should satisfy Condition L.

That the condition is necessary follows by an argument almost identical with part (II) of the proof of Theorem 1. I shall proceed to show that it is sufficient.

Proof I (depending on Theorem 4). Let $C$ denote a circle having center $O$ and not enclosing or containing any point of $M$. By an inversion of the plane about the circle $C, M$ is thrown into a bounded point set $M^{*}$ which is closed except possibly for the point $O$. I shall show that $M^{*}$ satisfies Condition L. Suppose the contrary is true; then there exists a continuum $K$ consisting of more than one point and such that for every positive number $\epsilon, K$ is the limiting set of a set of maximal connected subsets of $M^{*}$ each of diameter less than $\epsilon$. Let $P$ be a point of $K$ which is different from the point $O$, and let $J^{*}$ be a circle having $P$ as center and not containing or enclosing $O$. It is a consequence of Janiszewski's theorem $\dagger$ that $J^{*}$ plus its interior contains a subcontinuum $H^{*}$ of $K$ which consists of more than one point; $H^{*}$ does not contain $O$. Hence $H$, the point set of which $H^{*}$ is the image under this inversion, is a bounded point set. Let $I^{*}$ denote the interior of $J^{*}$, and let $J$ and $I$ denote the point sets of which $J^{*}$ and $I^{*}$ respectively are

† See Janiszewski, loc. cit. 
the images. Let $G_{1}^{*}$ denote a set of maximal connected subsets of $M^{*}$ each of which has a point within $J^{*}$ and is of diameter less than 1 and such that $H^{*}$ is a part of the limiting set of $G_{1}^{*}$; let $G_{2}{ }^{*}$ denote a corresponding set having $H^{*}$ as a part of its limiting set and such that each element of $G_{2}{ }^{*}$ has a point in $I^{*}$ and is of diameter less than $\frac{1}{2}$; let $G_{3}^{*}, G_{3}^{*}, \cdots$ denote corresponding sets for the numbers $\frac{1}{3}, \frac{1}{4}, \cdots$. Let $G_{1}, G_{2}, G_{3}, \cdots$ denote the point sets of which $G_{1}^{*}, G_{2}^{*}, G_{3}^{*}, \cdots$ are the images. Then $H$ is a part of the limiting set of each of the sets $G_{1}, G_{2}, G_{3}, \cdots$. But since $M$ satisfies Condition L, there exists a positive number $\epsilon_{H}$ such that $H$ is not the limiting set of any set of maximal connected subsets of $M$ each of diameter less than $\epsilon_{H}$. Then for every positive integer $n, G_{n}$ must contain at least one element $g_{n}$ which is of diameter $\geqq \epsilon_{H}$. From each set $G_{i}$, select one such element $g_{i}$, and let $B$ denote the sequence of sets $g_{1}, g_{2}, g_{3}, \cdots$ thus obtained. Since every element of $B$ contains at least one point in the bounded point set $I$, it follows that the sequence $B$ contains some subsequence $A$ which has a sequential limiting set $\bar{g}$ which is of diameter $\geqq \epsilon_{H}$. But $A^{*}$, the image of $A$, has the property that for every positive number $\epsilon$, there are not more than a finite number of elements of $A^{*}$ of diameter greater than $\epsilon$. Hence, the limiting set $\bar{g}^{*}$ of $A^{*}$ must consist of only a single point; but $\bar{g}^{*}$ is the image of $\bar{g}$, a point set of diameter $\geqq \epsilon_{H}$. Thus the supposition that $M^{*}$ does not satisfy Condition L leads to a contradiction. Then since $M^{*}$ satisfies Condition $\mathrm{L}$ and lacks only the point $O$ of being closed, it follows by Theorem 4 that there exists a simple continuous arc $t$ which contains at least one point of every maximal connected subset of $M^{*}$. Let $X$ and $Y$ denote the extremities of $t$. There exists an $\operatorname{arc} t_{0}$ from $X$ to $Y$ which has only the points $X$ and $Y$ in common with $t$. Let $l^{*}$ denote the simple closed curve $t+t_{0}$. Now if $l^{*}$ contains the point $O$, it can readily be shown that the point set of which $l^{*}-O$ is the image is an open curve which contains at least one point of every maximal connected subset of $M$. In case $l^{*}$ does not contain $O$, then $M$ must satisfy all the conditions of Theorem 2, and with the aid of that theorem, Theorem 6 can easily be established in this case.

Proof II (depending on Theorem 5). I will indicate how the condition may be proved sufficient using methods very similar to those used in the proof of Theorem 1. For any definite positive integer $n$, let the whole plane be divided by a countable infinity of horizontal and vertical straight lines into a countable number of squares plus their interiors in such a way that the interiors of no two of these squares have a point in common and so that the diameter of each of them is less than $1 / n$. Let $G$ denote this countable set of squares (not including their interiors) and let $T$ denote the point set obtained by adding together all the point sets of the set $G$. Let $K$ denote 
the point set common to $T$ and $M$. From each maximal connected subset $Y$ of $K$, select exactly one point $X$. Let $P$ denote the set of all such points $(X)$ thus selected. It can readily be shown, then, that $\bar{P}$ is a closed and totally disconnected point set. Then from Theorem 5 it follows that there exists an open curve $l_{n}$ which contains $\bar{P} ; l_{n}$ contains at least one point of every maximal connected subset of $M$ which is of diameter greater than $1 / n$. Hence, there exists a countable number of open curves $l_{1}, l_{2}, l_{3}, \ldots$, such that for every positive integer $n, l_{n}$ contains at least one point of every maximal connected subset of $M$ which is of diameter greater than $1 / n$. Then by an argument almost identical with that used in the proof of Theorem 1, using this countable set of open curves instead of a countable set of arcs as in that case, it follows that $M$ contains a closed and totally disconnected point set $R$ which contains at least one point of every maximal connected subset of $M$. Then, by Theurem 5 , there exists an open curve $l$ which contains $R ; l$ satisfies all the conditions of the open curve required in the statement of Theorem 6 .

THEOREM 7. If $M$ is any continuum whatever, and $K$ is a closed and totally disconnected subset of $M$, then the point set $M-K$ satisfies Condition $\mathrm{L}$.

Suppose $M-K$ does not satisfy Condition $L$. Then there exists a continuum $H$ consisting of more than one point such that for every positive number $\epsilon, H$ is a part of the limiting set of a set of maximal connected subsets of $M-K$ each of diameter less than $\epsilon$. Since $K$ is totally disconnected, there exists a point $P$ of $H$ which does not belong to $K$. Let $C$ be a circle having $P$ as center and not enclosing or containing any point of $K$. Let $r$ denote the radius of $C$. Let $N$ denote a set of maximal connected subsets of $M-K$ each of diameter less than $\frac{1}{4} r$, which has $H$ as a part of its limiting set. Then some element $g$ of $N$ contains a point $Q$ whose distance from $P$ is less than $\frac{1}{r} r$; and since $g$ is of diameter less than $\frac{1}{4} r, \bar{g}$ must lie wholly within $C$. But $\bar{g}$ has a point in $K,{ }^{*}$ and $C$ encloses no point of $K$. Thus the supposition that $M-K$ does not satisfy Condition $\mathrm{L}$ leads to a contradiction.

THEOREM 8. If $M$ is any continuum whatever, and $K$ is a bounded [unbounded], closed, and totally disconnected subset of $M$, then there exists an arc [open curve] which contains at least one point of every maximal connected subset of the point set $M-K$.

\footnotetext{
* By virtue of the following theorem: If $K$ is any closed subset of a continuum $M$ and $g$ is any bounded maximal connected subset of $M-K$, then $K$ contains at least one point of $g$.

UnIVERSiTy OF TEXas, Austin, Texas
} 